\title{
Desarrollo de la comprensión oral del idioma inglés a través del aprendizaje combinado B-learning en estudiantes universitarios
}

\author{
Development of oral comprehension of the English language \\ through the B-learning blended learning in university students
}

\author{
Marjorie Andrade Velásquez
}

ece@pucesd.edu.ec

\begin{abstract}
Resumen
La presente investigación propone el uso de la modalidad B-learning que combina la educación presencial y a distancia, retomando las ventajas de ambas modalidades como complemento y apoyo en el desarrollo de la comprensión oral de la asignatura de inglés de los estudiantes del cuarto nivel de la Pontificia Universidad Católica Sede Santo Domingo. Se utilizó para ésta investigación Dokeos, que es una plataforma educativa que brinda la capacidad de interactuar con uno o varios usuarios con fines pedagógicos. El diseño aplicado fue cuasi experimental pre-post, en un grupo intacto de cuarenta y seis estudiantes del idioma Inglés. Los resultados obtenidos revelan un mayor incremento en el aprendizaje, muestran que la mayoría de los estudiantes se sienten motivados con el uso de las tecnologías y observan de forma positiva la metodología B-learning como apoyo a las clases presenciales.
\end{abstract}

\section{Palabras claves}

Comprensión oral, metodología B-learning, educación presencial, educación a distancia.

\begin{abstract}
This research proposes the use of B-learning mode which combines classroom and distance education, retaking the advantages of both modalities to complement and support the development of oral comprehension of the English class students of the fourth level at Catholic University in Santo Domingo. Dokeos, was used for this research which is an educational platform that provides the ability to interact with one or more users for educational purposes. The quasi-experimental design was applied pre - post in an intact group of fortysix students of English. The results show a greater increase in learning, show that most of the students are motivated to use technology positively and observed B -learning methodology to support campus classes.
\end{abstract}

\section{Keywords}

Oral comprehension, B-learning modality, in-person education, distance education.

Forma sugerida de citar:

Andrade Velásquez, Marjorie (2015). Desarrollo de la comprensión oral del idioma inglés a través del aprendizaje combinado B-learning en estudiantes universitarios. Universitas, XIII(23), pp. 109-124. 


\section{Introducción}

Uno de los grandes desafíos que tienen los maestros es el nuevo rol y desempeño docente con el uso de las nuevas tecnologías de la información. Zambrano (2012) afirma que el uso de las tecnologías en educación, ha sido quizás el cambio y el reto más brusco y amplio en los últimos años y nos ofrecen la oportunidad de acceder a aulas virtuales que son importantes herramientas en la transformación de la educación y en los rápidos cambios que están tomando lugar en los entornos de aprendizaje en red. "Es necesario considerar las posibilidades que ofrecen las nuevas tecnologías, para crear ambientes virtuales, que favorezcan la comunicación bidireccional" (Silva Quiroz, 2011).

En los últimos años, las aulas virtuales tienen eficacia en la asignación de tareas, foros, seguimiento académico de los alumnos, evaluaciones en línea, entre otros, donde tanto alumnos como profesores e investigadores pueden acceder sin mayores limitaciones de tiempo y espacio y ha producido cambios en la no utilización de materiales impresos, guías de estudios, de trabajo o laboratorio.

Esta investigación nació de la inquietud de la autora al observar que los estudiantes de inglés de la Pontificia Universidad Católica Sede Santo Domingo, al terminar los cursos aún tienen dificultad para comunicarse en el idioma meta. Esta idea se confirmó al realizar algunas observaciones y al escuchar los comentarios de los maestros, quienes decían que los estudiantes no aprenden a comunicarse oralmente, y por su lado los estudiantes mencionaban la dificultad de poder interactuar en este idioma, debido a factores como la falta de tiempo en las horas de clases, para la práctica adecuada de la destreza oral y la desmotivación de los mismos.

La comprensión oral es un proceso complejo si se tienen en cuenta todos los mecanismos involucrados. El acto de la comunicación empieza en la mente del hablante, gracias a la función creativa, se concibe y se forma el mensaje. Esto es posible gracias al conocimiento profundo del funcionamiento del lenguaje almacenado en la mente (Corpas \& Madrid, 2009).

La comprensión oral es una actividad que admite el intercambio de información desde el comienzo de la vida. Los estudiantes para estudiar una lengua primero deben aprender a desarrollar la habilidad receptiva del idioma que es escuchar y luego aprende hablar, leer y escribir.

El B-learning o Blended Learning es una modalidad semipresencial que ayuda en el proceso de aprendizaje de los estudiantes en un ambiente de aprendizaje combinado B-learning, en el que se mezcla diferentes metodologías de 
enseñanza-aprendizaje, incluyendo las clases presenciales, así como las clases no presenciales, en la que los estudiantes pueden reforzar y ejercitar de manera autónoma los contenidos revisados en clases y a la vez utilizar herramientas de comunicación y de trabajo colaborativo como aporte para el desarrollo de la comprensión oral del idioma inglés.

La actualización profesional en el mundo de la formación es fundamental, ya que el conocimiento y las nuevas formas de enseñar no dejan de evolucionar, de ahí la importancia de adquirir competencias para desarrollar la tarea de educador B - learning, una profesión con demanda en el mercado laboral y que tiene una gran proyección de futuro.

\section{Metodología}

Su objetivo fue desarrollar la comprensión oral del inglés a través de la metodología B-learning en cuarenta y seis estudiantes de cuarto nivel de inglés de la PUCESD y utilizando la Plataforma Dokeos como medio para las clases no presenciales. Los estudiantes recibieron instrucción explícita acerca del uso de la Plataforma, durante el desarrollo de sus clases de inglés, por un lapso de ocho semanas, sin contar la aplicación del pretest y postest.

Con el fin de verificar las hipótesis, se analizaron los resultados obtenidos por estudiantes universitarios cuarto nivel de inglés en las pruebas pre y postest diseñadas de acuerdo a las estándares para la evaluación de la comprensión oral del inglés, según el Marco Común Europeo de Referencia: A1.1, A1.2, A2.1, A2.2, respectivamente. Al inicio el nivel de dominio de los sujetos estaba relacionado con el curso que estudiaban al momento de la intervención.

Además de las pruebas pre-post, se aplicó una encuesta antes y después de la aplicación de la nueva metodología para indagar acerca de las reflexiones y sugerencias de los estudiantes en torno a las tareas de audición, materiales utilizados y el uso de la nueva metodología B-learning.

\section{Resultados}

Los resultados principales del estudio se pueden agrupar en diferentes categorías: El nivel alcanzado dentro del Marco Común Europeo, la percepción positiva del curso, el nivel de satisfacción de los estudiantes y la opinión favorable de los docentes del área de Inglés sobre el Blended Learning como modalidad 
de apoyo en el aprendizaje del inglés y la mezcla de dos procesos de aprendizaje, este punto coincide con la opinión de Quiroz (2011) "Lo virtual se complementa con lo presencial y viceversa y se conjugan las características de ambos procesos de enseñanza" (p.39).

De la observación de los métodos y técnicas actuales utilizadas en las clases tradicionales del inglés, se puede observar que cada docente trabaja con metodologías diferentes, pero el tiempo en las horas de clases no es suficiente para desarrollar las competencias comunicativas en su totalidad, por lo que se recomienda la oportunidad de disponer de un medio tecnológico diseñado de acuerdo al desarrollo de cada una de las destrezas del idioma, donde el docente podrá ofrecer actividades y tiempo extra

A través de la evaluación de la funcionalidad del curso y los impactos, teniendo en cuenta aquellas necesidades de la misma, interés, conocimientos previos y criterios de los estudiantes previo a la creación de cada usuario en la plataforma Dokeos. La encuesta propuesta arrojó datos cuantitativos y cualitativos los mismos que permitieron demostrar mediante gráficas realizadas la aceptación del proceso, la funcionalidad y satisfacción de la metodología de enseñanza y los impactos del curso. En general los estudiantes afirmaron sentirse motivados, los contenidos del curso fueron adecuados y la metodología B-learning, ha permitido el desarrollo de la comprensión oral del idioma inglés y a la vez tiene un efecto motivacional en el estudiante, lo cual coadyuva al logro de los resultados de aprendizaje (Ausubel, Novak y Hanesian, 2005).

Dentro de la investigación se consideró una variable independiente y una dependiente, como variable dependiente se consideró el desarrollo de la comprensión oral del inglés y la variable independiente el curso B-learning. Es importante mencionar que la construcción del pre y post test se elaboró de acuerdo a los parámetros considerados para evaluar la destreza oral del idioma inglés como son: pronunciación, ritmo y entonación, vocabulario, gramática, fluidez y comprensión auditiva.

Los resultados de las pruebas pre y post aplicada a los estudiantes al inicio y al final del curso, fueron clasificados en base a la tabla de acuerdo al Marco Común Europeo de referencia, el mismo que establece una serie de niveles para todas las lenguas a partir de los cuales se favorece la comparación u homologación de los distintos títulos emitidos por las entidades certificadas. 


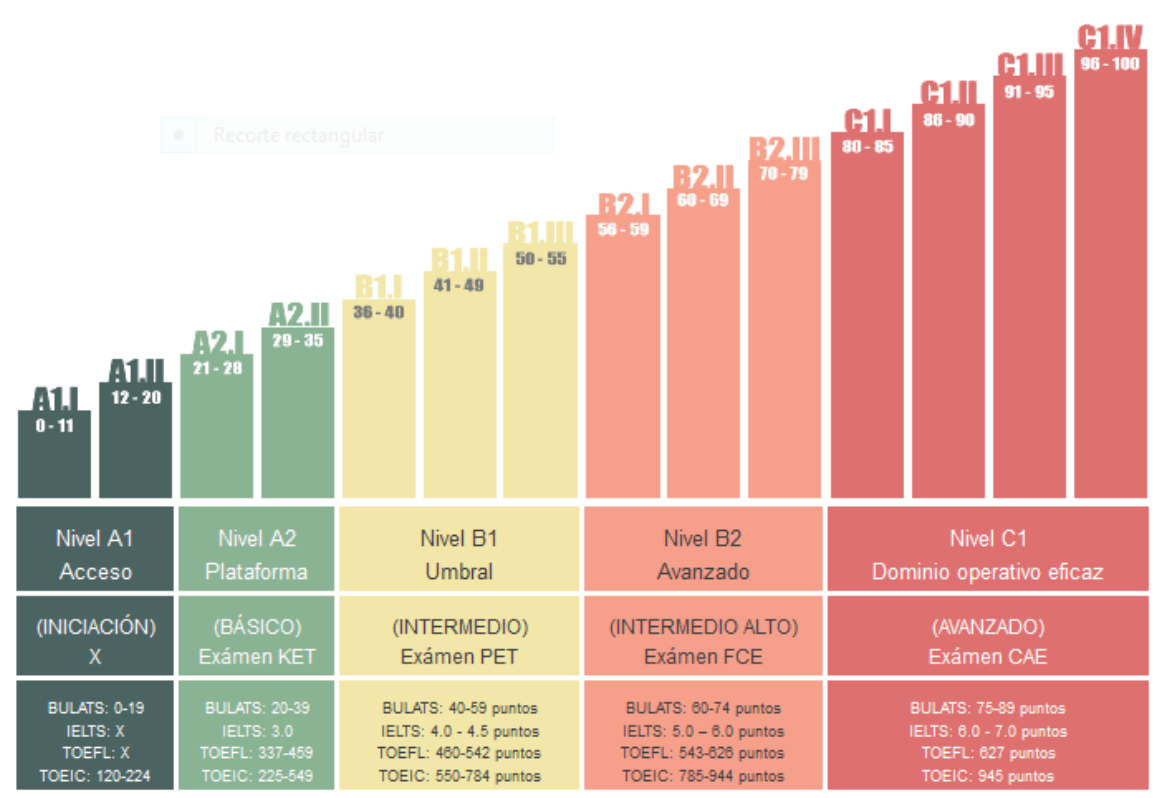

De 0 a 11 nivel A1.1 Usuario iniciación

De 12 a 20 nivel A1.2 Usuario iniciación

De 21 a 28 nivel A2.1 Usuario básico

De 29 a 35 nivel A2.2 Usuario básico

De 36 a 40 nivel B1.1 Usuario intermedio

De 41 a 49 nivel B1.2 Usuario intermedio

Con el puntaje adquirido por cada estudiante, en las pruebas pre post se confirma mejores resultados que la modalidad a distancia y la modalidad tradicional, ya que de este modo se combinaron aspectos presenciales y virtuales de manera tal que los estudiantes concurrían a una clase semanal y trabajaban y se comunicaban en forma virtual con profesores, tutores y expertos de diferentes medios, enmarcando una propuesta multidireccional interactiva, que intentó promover el aprendizaje autónomo, apoyado por una organización tutorial y que utilizó los medios y recursos técnicos, para el logro de los objetivos propuestos como lo confirman (Loyo \& Rivero de Magnago, 2005).

Como se aprecia en la tabla 1, existe una diferencia en el porcentaje de los resultados de la pruebas pre-post. Al someter los datos a la prueba estadística 
de Wilcoxon se confirmó que como la menor de las sumas de los rangos es 0 y $0<195$, la decisión es rechazar la hipótesis nula.

\section{Tabla 1}

\section{Porcentajes pruebas pre-post test estudiantes cuarto nivel PUCE SD}

\begin{tabular}{|l|l|}
\hline PRE - TEST & $12.00 \%$ \\
\hline POST-TEST & $14.67 \%$ \\
\hline DIFERENCIA & $2.67 \%$ \\
\hline
\end{tabular}

Fuente: Pre y Post-Test

Elaboración: Marjorie Andrade Velásquez

El grupo de estudiantes en general presentaron porcentajes similares al momento de rendir el pre-test. El 80\% de los estudiantes en general, tenía un nivel de inglés de acuerdo al Marco Común Europeo A11. En las ocho semanas que se trabajó con la implementación del curso B- learning, y al aplicar el post-test, los estudiantes presentaron porcentajes mayores de conocimientos y desarrollo de la comprensión oral.

La Tabla 2 y 3 representan los resultados observados por habilidad en el posttest del grupo intacto de 46 estudiantes. Se puede señalar el avance total en la comprensión auditiva y producción oral luego de las 8 semanas. Con la suma total de los puntajes obtenidos por cada uno de los estudiantes, se realizó la clasificación de niveles de acuerdo a la tabla del Marco Común Europeo de Referencia.

Tabla 2

Resultados pre-test estudiantes cuarto nivel Pontificia Universidad Católica Sede Santo Domingo

\begin{tabular}{|l|c|c|c|l|}
\hline $\mathbf{N}^{\mathbf{o}}$ estudiante & $\begin{array}{c}\text { Comprensión } \\
\text { Auditiva }\end{array}$ & Producción Oral & Total & Nivel \\
\hline 1 & 18 & 10 & 28 & A21 \\
\hline 2 & 15.24 & 16 & 31.24 & A22 \\
\hline 3 & 16.08 & 10.5 & 26.58 & A21 \\
\hline 4 & 13.65 & 10.33 & 23.98 & A21 \\
\hline 5 & 8.84 & 9.67 & 18.51 & A12 \\
\hline
\end{tabular}




\begin{tabular}{|c|c|c|c|c|}
\hline $\mathbf{N}^{0}$ estudiante & $\begin{array}{c}\text { Comprensión } \\
\text { Auditiva }\end{array}$ & Producción Oral & Total & Nive \\
\hline 6 & 12.34 & 12.45 & 24.79 & $\mathrm{~A} 21$ \\
\hline 7 & 15.6 & 12 & 27.6 & A21 \\
\hline 8 & 12 & 15 & 27 & $\mathrm{~A} 21$ \\
\hline 9 & 11.35 & 16.65 & 28 & A21 \\
\hline 10 & 8.94 & 7.66 & 16.6 & A12 \\
\hline 11 & 12.88 & 11.95 & 24.83 & A21 \\
\hline 12 & 13.35 & 11.49 & 24.84 & A21 \\
\hline 13 & 10.4 & 11.28 & 21.68 & A21 \\
\hline 14 & 12.35 & 13.25 & 25.6 & $\mathrm{~A} 21$ \\
\hline 15 & 13.03 & 14.46 & 27.49 & $\mathrm{~A} 21$ \\
\hline 16 & 14.72 & 15.25 & 29.97 & A22 \\
\hline 17 & 10.72 & 11.57 & 22.29 & A21 \\
\hline 18 & 15 & 12 & 27 & $\mathrm{~A} 21$ \\
\hline 19 & 9.62 & 9 & 18.62 & A12 \\
\hline 20 & 13.34 & 12 & 25.34 & A21 \\
\hline 21 & 11.12 & 12.1 & 23.22 & $\mathrm{~A} 21$ \\
\hline 22 & 16.85 & 10.75 & 27.6 & A21 \\
\hline 23 & 12.31 & 11.55 & 23.86 & A21 \\
\hline 24 & 10.09 & 10.23 & 20.32 & A12 \\
\hline 25 & 10.96 & 12.75 & 23.71 & A21 \\
\hline 26 & 10.22 & 11.22 & 21.44 & A21 \\
\hline 27 & 11.91 & 12.36 & 24.27 & $\mathrm{~A} 21$ \\
\hline 28 & 13.96 & 13.61 & 27.57 & A21 \\
\hline 29 & 9.3 & 8.5 & 17.8 & A12 \\
\hline 30 & 9.56 & 9.69 & 19.25 & A12 \\
\hline 31 & 10.77 & 18.52 & 29.29 & A22 \\
\hline 32 & 10.09 & 12 & 22.09 & A21 \\
\hline 33 & 13.64 & 11.14 & 24.78 & A21 \\
\hline 34 & 12.35 & 13.27 & 25.62 & A21 \\
\hline 35 & 12.52 & 13.46 & 25.98 & A21 \\
\hline 36 & 11.54 & 10.38 & 21.92 & A21 \\
\hline
\end{tabular}




\begin{tabular}{|l|c|c|c|l|}
\hline $\mathbf{N}^{\mathbf{0}}$ estudiante & $\begin{array}{c}\text { Comprensión } \\
\text { Auditiva }\end{array}$ & Producción Oral & Total & Nivel \\
\hline 37 & 12.08 & 12.36 & 24.44 & A21 \\
\hline 38 & 16.59 & 11.24 & 27.83 & A21 \\
\hline 39 & 13.24 & 16.23 & 29.47 & A22 \\
\hline 40 & 12 & 11 & 23 & A21 \\
\hline 41 & 10 & 9 & 19 & A12 \\
\hline 42 & 10.23 & 11.82 & 22.05 & A21 \\
\hline 43 & 11.43 & 10.89 & 22.32 & A21 \\
\hline 44 & 9.32 & 10.45 & 19.77 & A12 \\
\hline 45 & 7.94 & 10.45 & 18.39 & A12 \\
\hline 46 & 8.91 & 10.48 & 19.39 & A12 \\
\hline
\end{tabular}

Fuente: Prueba pre test aplicada a los estudiantes de cuarto nivel de la PUCE SD.

Elaboración: Marjorie Andrade Velásquez.

Tabla 3

Resultados post-test estudiantes cuarto nivel de la PUCE SD

\begin{tabular}{|l|c|c|c|l|}
\hline $\mathbf{N}^{\mathbf{0}}$ estudiante & $\begin{array}{c}\text { Comprensión } \\
\text { auditiva }\end{array}$ & Producción oral & Total & Nivel \\
\hline 1 & 19 & 11 & 30 & A22 \\
\hline 2 & 17.34 & 17.8 & 35.14 & A22 \\
\hline 3 & 17.5 & 13.8 & 31.3 & A22 \\
\hline 4 & 17.55 & 12.33 & 29.88 & A22 \\
\hline 5 & 10.87 & 10.67 & 21.54 & A21 \\
\hline 6 & 19.5 & 18.5 & 38 & B11 \\
\hline 7 & 16 & 13.6 & 34.6 & B11 \\
\hline 8 & 16.5 & 18.1 & 32.35 & A22 \\
\hline 9 & 15.35 & 17 & 36 & B11 \\
\hline 10 & 18 & 18 & 29.5 & A22 \\
\hline 11 & 14.5 & 15 & 36 & B11 \\
\hline 12 & 17.5 & 18.5 & 24.78 & A21 \\
\hline 13 & 12.48 & 12.3 & 29.5 & A22 \\
\hline 14 & 14 & 15.5 & 31.06 & A22 \\
\hline 15 & 15.6 & & & \\
\hline
\end{tabular}




\begin{tabular}{|c|c|c|c|c|}
\hline 16 & 17 & 17.25 & 34.25 & A 22 \\
\hline 17 & 16 & 18 & 34 & A 22 \\
\hline 18 & 18.7 & 12 & 30.7 & A22 \\
\hline 19 & 16.3 & 19 & 35.3 & A22 \\
\hline 20 & 18.5 & 18 & 36.5 & B11 \\
\hline 21 & 14 & 15 & 29 & A22 \\
\hline 22 & 17.3 & 12 & 29.3 & A 22 \\
\hline 23 & 16.4 & 14 & 30.4 & A 22 \\
\hline 24 & 13 & 12.23 & 25.23 & A21 \\
\hline 25 & 15 & 15 & 30 & A22 \\
\hline 26 & 12 & 14 & 26 & A 21 \\
\hline 27 & 16 & 14 & 30 & A 22 \\
\hline 28 & 13.2 & 14.5 & 27.7 & A21 \\
\hline 29 & 13 & 11.58 & 24.58 & A 21 \\
\hline 30 & 11.6 & 12.69 & 24.29 & A 21 \\
\hline 31 & 12 & 19 & 31 & A 22 \\
\hline 32 & 13.7 & 15.6 & 29.3 & A22 \\
\hline 33 & 12 & 13.5 & 25.5 & A21 \\
\hline 34 & 13.5 & 16 & 29.5 & A22 \\
\hline 35 & 15.3 & 15.46 & 30.76 & A22 \\
\hline 36 & 14 & 10.5 & 24.5 & A 21 \\
\hline 37 & 15.3 & 16 & 31.3 & A22 \\
\hline 38 & 14.7 & 13 & 27.7 & A21 \\
\hline 39 & 14.9 & 14.23 & 29.13 & A 22 \\
\hline 40 & 10.1 & 11 & 21.1 & A 21 \\
\hline 41 & 17 & 14 & 31 & A22 \\
\hline 42 & 11.8 & 13 & 24.8 & A 21 \\
\hline 43 & 16.7 & 12 & 28.7 & A 22 \\
\hline 44 & 11.7 & 14 & 25.7 & A 21 \\
\hline 45 & 10 & 12.5 & 22.5 & A 21 \\
\hline 46 & 11 & 10.5 & 21.5 & A21 \\
\hline
\end{tabular}

Fuente: Prueba post aplicada a los estudiantes de cuarto nivel de la PUCE SD.

Elaboración: Marjorie Andrade Velásquez. 
La Tabla 4 representa los resultados totales del pre-y post-test y la diferencia alcanzada. Se puede señalar que el avance de cada estudiante en cuanto a los niveles de referencia del Marco Común Europeo en la mayoría de los estudiantes luego de las ocho semanas. De acuerdo con los datos presentados en las tablas.

\section{Tabla 5}

Diferencia Final entre pre- test y pos-test

\begin{tabular}{|c|c|c|c|c|c|}
\hline $\mathrm{N}^{0}$ estudiante & POST-TEST & PRE-TEST & Diferencia & MCE & MCE \\
\hline 1 & 30 & 28 & 2 & A21 & A22 \\
\hline 2 & 35.14 & 31.24 & 3.9 & A22 & A22 \\
\hline 3 & 31.3 & 26.58 & 4.72 & A21 & A22 \\
\hline 4 & 29.88 & 23.98 & 5.9 & A21 & A22 \\
\hline 5 & 21.54 & 18.51 & 3.03 & A12 & A21 \\
\hline 6 & 38 & 24.79 & 13.21 & A21 & B11 \\
\hline 7 & 29.6 & 27.6 & 2 & $\mathrm{~A} 21$ & A22 \\
\hline 8 & 34.6 & 27 & 7.6 & $\mathrm{~A} 21$ & B11 \\
\hline 9 & 32.35 & 28 & 4.35 & A21 & A22 \\
\hline 10 & 36 & 16.6 & 19.4 & A12 & B11 \\
\hline 11 & 29.5 & 24.83 & 4.67 & A21 & A22 \\
\hline 12 & 36 & 24.84 & 11.16 & A21 & B11 \\
\hline 13 & 24.78 & 21.68 & 3.1 & A21 & A21 \\
\hline 14 & 29.5 & 25.6 & 3.9 & A21 & A22 \\
\hline 15 & 31.06 & 27.49 & 3.57 & A21 & A22 \\
\hline 16 & 34.25 & 29.97 & 4.28 & A22 & A22 \\
\hline 17 & 34 & 22.29 & 11.71 & A21 & A22 \\
\hline 18 & 30.7 & 27 & 3.7 & A21 & A22 \\
\hline 19 & 35.3 & 18.62 & 16.68 & A12 & A22 \\
\hline 20 & 36.5 & 25.34 & 11.16 & A21 & B11 \\
\hline 21 & 29 & 23.22 & 5.78 & A21 & A22 \\
\hline 22 & 29.3 & 27.6 & 1.7 & A21 & A22 \\
\hline 23 & 30.4 & 23.86 & 6.54 & A21 & A22 \\
\hline 24 & 25.23 & 20.32 & 4.91 & A12 & A21 \\
\hline 25 & 30 & 23.71 & 6.29 & A21 & A22 \\
\hline
\end{tabular}




\begin{tabular}{|l|c|c|c|l|l|}
\hline $\mathbf{N}^{\mathbf{0}}$ estudiante & POST-TEST & PRE-TEST & Diferencia & \multicolumn{1}{|c|}{ MCE } & \multicolumn{1}{|c|}{ MCE } \\
\hline 26 & 26 & 21.44 & 4.56 & A21 & A21 \\
\hline 27 & 30 & 24.27 & 5.73 & A21 & A22 \\
\hline 28 & 27.7 & 27.57 & 0.13 & A21 & A21 \\
\hline 29 & 24.58 & 17.8 & 6.78 & A12 & A21 \\
\hline 30 & 24.29 & 19.25 & 5.04 & A12 & A21 \\
\hline 31 & 31 & 29.29 & 1.71 & A22 & A22 \\
\hline 32 & 29.3 & 22.09 & 7.21 & A21 & A22 \\
\hline 33 & 25.5 & 24.78 & 0.72 & A21 & A21 \\
\hline 34 & 29.5 & 25.62 & 3.88 & A21 & A22 \\
\hline 35 & 30.76 & 25.98 & 4.78 & A21 & A22 \\
\hline 36 & 24.5 & 21.92 & 2.58 & A21 & A21 \\
\hline 37 & 31.3 & 24.44 & 6.86 & A21 & A22 \\
\hline 38 & 27.7 & 27.83 & -0.13 & A21 & A21 \\
\hline 39 & 29.13 & 29.47 & -0.34 & A22 & A22 \\
\hline 40 & 21.1 & 23 & -1.9 & A21 & A21 \\
\hline 41 & 31 & 19 & 12 & A12 & A22 \\
\hline 42 & 24.8 & 22.05 & 2.75 & A21 & A21 \\
\hline 43 & 28.7 & 22.32 & 6.38 & A21 & A22 \\
\hline 44 & 25.7 & 19.77 & 5.93 & A12 & A21 \\
\hline 45 & 22.5 & 18.39 & 4.11 & A12 & A21 \\
\hline 46 & 19.39 & 2.11 & A12 & A21 \\
\hline
\end{tabular}

\subsection{5/50 24/50}

Fuente: Prueba pre-post aplicada a los estudiantes de cuarto nivel de la PUCE SD.

Elaboración: Marjorie Andrade Velásquez. 


\section{Gráfico 2}

\section{Diferencia nota Pre-Post test estudiantes cuarto nivel de la PUCE SD}

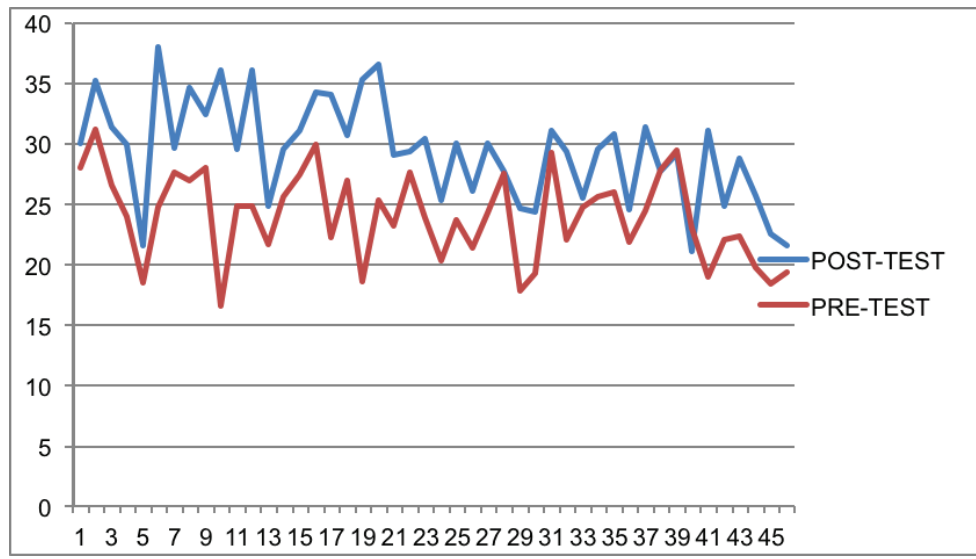

Fuente: Prueba pre-post aplicada a los estudiantes de cuarto nivel de la PUCE SD.

Elaboración: Marjorie Andrade Velásquez

Basados en los resultados de este estudio y aplicando la tabla estadística de Wilcoxon, se puede decir que el modelo de Aprendizaje Combinado o modalidad semi presencial se presenta como una metodología válida para sustentar la enseñanza de idiomas extranjeros. Por lo tanto, sugerimos que los profesores de inglés deben incorporar en sus cursos modelos de aprendizaje que incluyan clases presenciales y ndo presenciales, apoyadas en el uso de las distintas tecnologías de información y comunicación.

Tabla 6

Aplicación de la Prueba de rangos con signo de Wilcoxon

\begin{tabular}{|l|l|l|l|l|l|l|l|l|l|}
\cline { 3 - 8 } \multicolumn{1}{l|}{} & \multicolumn{3}{c}{ Resultados totales } & \multicolumn{3}{c|}{$\begin{array}{c}\text { Rangos } \\
\text { asignados }\end{array}$} & \multicolumn{2}{c|}{} \\
\hline Estudiante & Post-test & Pre-test & $\begin{array}{c}\text { Diferencia en } \\
\text { la calificación }\end{array}$ & $\begin{array}{c}\text { Diferencia } \\
\text { absoluta }\end{array}$ & Rango & R+ & R- & & \\
\hline 1 & 30,00 & 28,00 & 2,00 & 2 & 4 & 4 & & & \\
\hline 2 & 35,14 & 31,24 & 3,90 & 3,9 & 12 & 12 & & & \\
\hline 3 & 31,30 & 26,58 & 4,72 & 4,72 & 17 & 17 & & & \\
\hline
\end{tabular}


Marjorie Andrade Velásquez. Desarrollo de la comprensión oral del idioma inglés

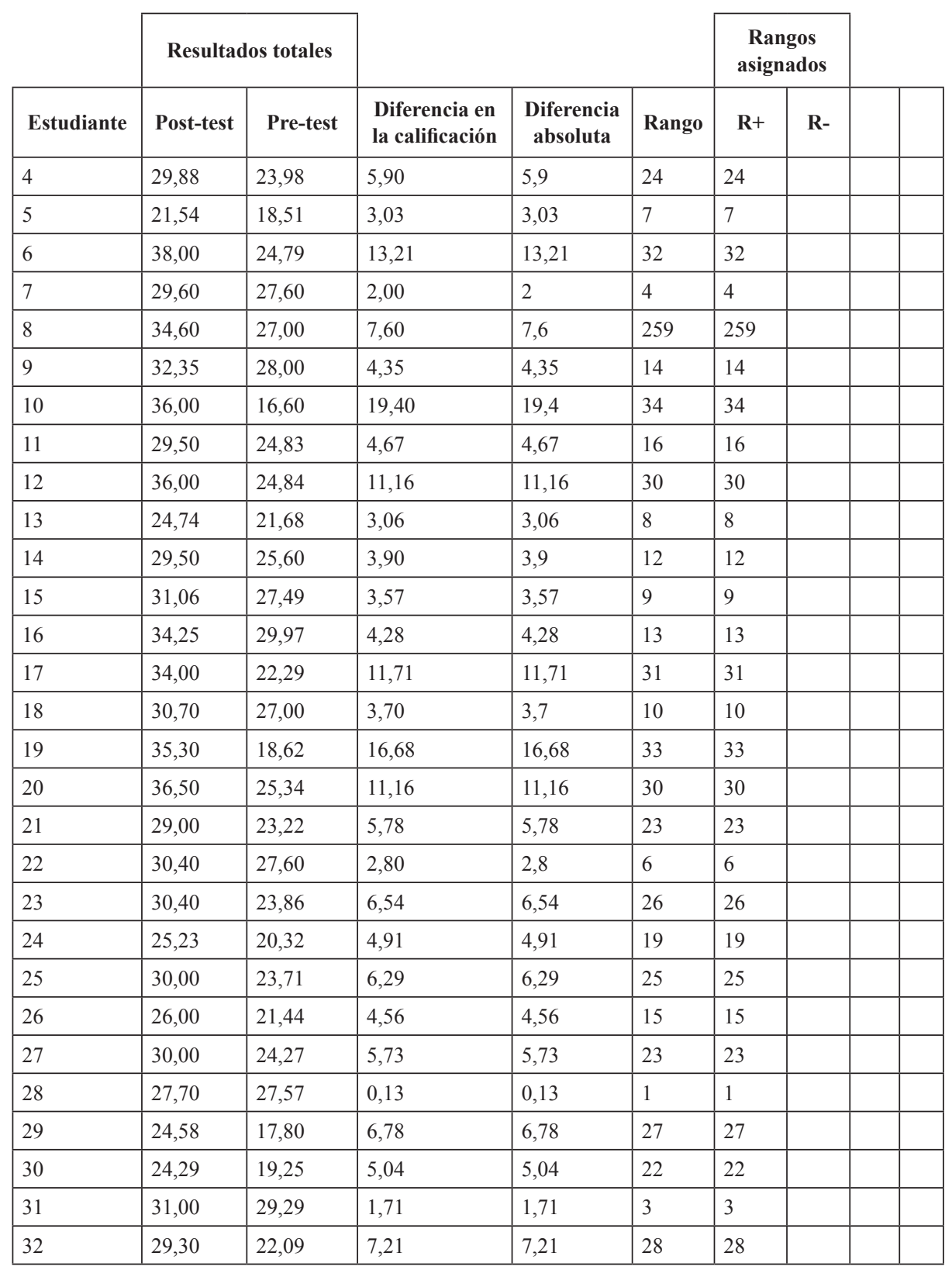




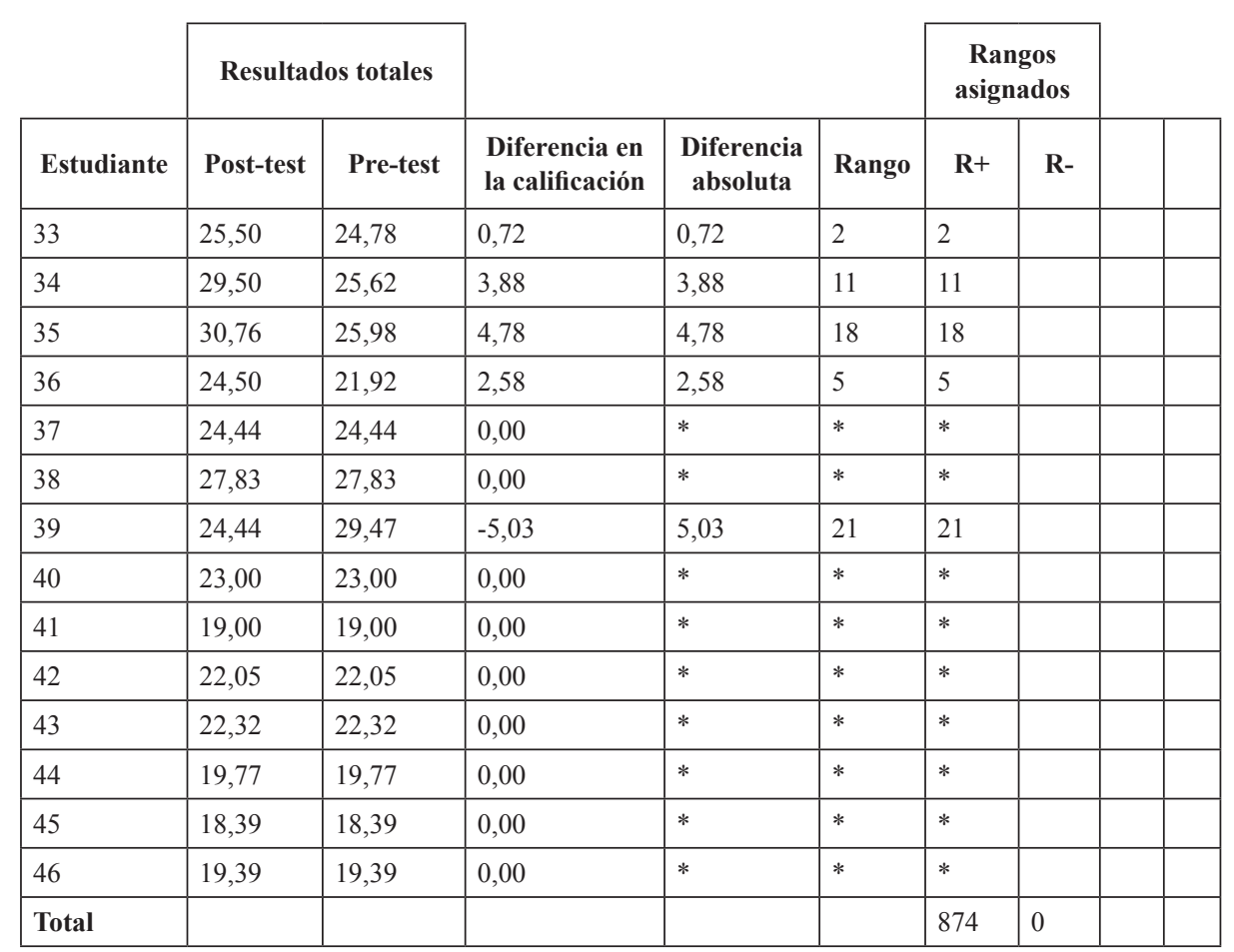

\section{Planteamiento de la hipótesis}

Ho: No existe diferencia significativa entre el rendimiento académico de los estudiantes en las pruebas pre y post test después de haber sido aplicada la nueva metodológica. 106

Ho: $\mu \mathbf{x}-\mu \mathbf{y}=\mathbf{0}$

H1: existe diferencia significativa entre el rendimiento académico de los estudiantes en las pruebas pre y post test después de haber sido aplicada la nueva metodológica.

H1: $\mu \mathbf{x}-\mu \mathbf{x} \neq \mathbf{0}$

\section{Nivel de significancia}

Se asigna esta prueba de hipótesis con un nivel de significancia $\square=5 \%$ con $n=37$ obteniendo el valor crítico de la tabla $\mathrm{T}$ de Wilcoxon $=195$.

\section{Descripción de la población y suposiciones}

En este caso, la muestra está constituida por los 46 estudiantes de cuarto nivel de la Pontificia Universidad Católica Sede Santo Domingo. Teniendo en cuenta que 9 estudiantes calificaron con igual puntaje en sus dos pruebas y se eliminan del estudio, haciendo que ahora el tamaño de la muestra sea de $46-9=37$.

Fuente: Tabla de rangos con signo de Wilcoxon.

Elaboración: Marjorie Andrade Velásquez 
Regla de decisión: La regla de decisión será rechazar la hipótesis nula si el menor de la sumas de rangos es menor o igual a 195.

\section{Decisión estadística}

Como la menor de las sumas de los rangos es 0 y $0<195$, la decisión es rechazar la hipótesis nula. El grupo de estudiantes en general presentaron porcentajes similares al momento de rendir el pre - test. El 80\% de los estudiantes en general, tenía un nivel de inglés de acuerdo al Marco Común Europeo A11. En las ocho semanas que se trabajó con la implementación del curso B-learning y al aplicar el post-test los estudiantes presentaron porcentajes mayores de conocimientos y desarrollo de la comprensión oral. Sobre la base de las pruebas realizadas, se concluye que sí existe diferencia significativa en la comprensión oral del inglés de los estudiantes de antes de la aplicación de la metodología B- learning.

\section{Conclusiones}

- La utilización de la metodología B-learning incide en el rendimiento académico de los estudiantes de inglés de la Pontificia Universidad Católica Sede Santo Domingo.

- Los resultados obtenidos permiten corroborar que los estudiantes participantes incrementaron la comprensión oral del idioma inglés en un $2.67 \%$, el modelo de Aprendizaje Combinado o modalidad semipresencial B-learning se presenta como una metodología válida para el desarrollo de la comprensión oral. El desarrollo de la comprensión oral del idioma inglés se vio favorecido con las clases presenciales y no presenciales, apoyadas en el uso de las distintas tecnologías de la información y comunicación.

- El B-learning desarrolla y perfecciona nuevas estrategias de aprendizaje y a su vez favorece la autogestión ya que los alumnos pueden distribuir su tiempo a su ritmo y facilita la interacción, comunicación e integración más rápida y a su vez se convierten en protagonista de su aprendizaje, mediante el uso de estrategias autónomas de búsqueda y selección de información. 


\section{Bibliografía}

Bautista, G., Borges, F., \& Forés, A. (2006). Didáctica Universitaria en entornos virtuales de enseñanza - aprendizaje. Madrid: Ediciones Narcea.

Barkley, E. F. (2007). Técnicas de aprendizaje colaborativo: Manual para el profesorado universitario. Madrid: Ediciones Morata, S.L.

Loyo, A. C., \& Rivero de Magnago, M. (2005). Las lenguas extranjeras y las nuevas tecnologías de la comunicación. Argentina: Editorial Graó.

Martín Martín, J. M. (2000). La lengua materna en el aprendizaje de una segunda lengua. España: J.de Haro Artes Gráficas, S.L.P.I.S.A Sevilla.

Ministerio de Eduación y Ciencia. (2004). Nuevas formas de aprendizaje en Lenguas extranjeras. Estilo Estugrf Impresores S.I.

Murado Bouso, J. L. (2010). Didáctica de inglés en educación infantil. Métodos para la enseñanza y el aprendizaje de la dLengua Inglesa. España: Ideaspropias.

Nesterenko,N. (2008). Guía Didáctica: Methodology I. Loja-Ecuador: Editorial de la Universidad Técnica Particular de Loja.

Pavesi, M., Vertocchi, D., Hofmannová, M., \& Kazianka, M. (2001). Enseñar en una lengua extranjera, G. Langé (Ed.). Roma.

Richards, J. C., \& Rodgers, T. S. (2001). Approaches and Methods in Language Teaching. New York: Cambridge University Press.

Rodríguez Illera, J. L. (2008). Comunidades virtuales de práctica y de aprendizaje. España: Publications 1 Edicions de la Universitat de Barcelona.

Ruiz Velasco Sánchez, E. (2013). Tecnologías de la información y la educación para la innovación educativa. México, D. F.: Edicciones Díaz de Santos.

Silva Salinas, S. (2005). Medios Didácticos Multimedia para el aula. Lass NNTT como herramientas didácticas en los centros educativos. España: Ideas Propias Editorial.

Zambrano Ayala, W. (2012). Modelo de Aprendizaje Virtual para la Educación Superior MAVES basado en tecnologías Web 3.0. Bogotá: Ecoe Ediciones. 